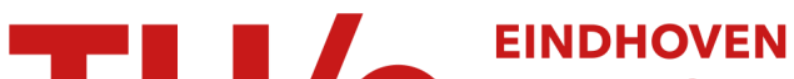 UNIVERSITY OF TECHNOLOGY
}

\section{Frequency selective surfaces for extended bandwidth backing reflector functions}

Citation for published version (APA):

Pasian, M., Monni, S., Neto, A., Ettorre, M., \& Gerini, G. (2010). Frequency selective surfaces for extended bandwidth backing reflector functions. IEEE Transactions on Antennas and Propagation, 58(1), 43-50.

https://doi.org/10.1109/TAP.2009.2036185

DOI:

10.1109/TAP.2009.2036185

Document status and date:

Published: 01/01/2010

Document Version:

Publisher's PDF, also known as Version of Record (includes final page, issue and volume numbers)

Please check the document version of this publication:

- A submitted manuscript is the version of the article upon submission and before peer-review. There can be important differences between the submitted version and the official published version of record. People interested in the research are advised to contact the author for the final version of the publication, or visit the $\mathrm{DOI}$ to the publisher's website.

- The final author version and the galley proof are versions of the publication after peer review.

- The final published version features the final layout of the paper including the volume, issue and page numbers.

Link to publication

\section{General rights}

Copyright and moral rights for the publications made accessible in the public portal are retained by the authors and/or other copyright owners and it is a condition of accessing publications that users recognise and abide by the legal requirements associated with these rights.

- Users may download and print one copy of any publication from the public portal for the purpose of private study or research.

- You may not further distribute the material or use it for any profit-making activity or commercial gain

- You may freely distribute the URL identifying the publication in the public portal.

If the publication is distributed under the terms of Article $25 \mathrm{fa}$ of the Dutch Copyright Act, indicated by the "Taverne" license above, please follow below link for the End User Agreement:

www.tue.nl/taverne

Take down policy

If you believe that this document breaches copyright please contact us at:

openaccess@tue.nl

providing details and we will investigate your claim. 


\title{
Frequency Selective Surfaces for Extended Bandwidth Backing Reflector Functions
}

\author{
Marco Pasian, Member, IEEE, Stefania Monni, Member, IEEE, Andrea Neto, Member, IEEE, Mauro Ettorre, and \\ Giampiero Gerini, Senior Member, IEEE
}

\begin{abstract}
This paper deals with the use of frequency selective surfaces (FSS) to increase the efficiency $\times$ bandwidth product in wideband antenna arrays, whose efficiency is limited by the front-to-back ratio. If the backing reflector for the antenna is realized through a single metal plane solution, its location will be suitable only on a relatively limited frequency range especially if wide angle scanning is required. In order to extend the frequency range of usability, an FSS can be sandwiched between the antenna and the ground plane, providing an additional reflecting plane for an higher frequency band. The possibility to integrate in the antenna different functionalities, otherwise performed by several antennas, is also discussed in the paper. The proposed backing structure composed by the FSS and the ground plane has been designed to be used in conjunction with a wideband antenna consisting of an array of connected dipoles. A hardware demonstrator of the backing structure has also been manufactured and tested.
\end{abstract}

Index Terms-Connected array, frequency selective surface, Green's function, wideband antenna, wide-scan antenna.

\section{INTRODUCTION}

C URRENT trends in the design of military ship masts foresee the integration of several functionalities on the same antenna aperture in order to satisfy the demand of an increasing number of services to be installed on board, while still responding to the requirement of reducing the radar cross-section (RCS) [1] of the ship itself. In view of this, multiband/broadband planar or quasi-planar antennas with large scanning capabilities are required.

Existing solutions, such as the Vivaldi antenna [2] show good performances at the cost of low cross polarization purity, which limits the range of possible applications. Other antennas, such as the connected array without a backing reflector, as described in [3], show excellent performances in terms of operating bandwidth, but an efficiency that can be as low as $50 \%$, because of the poor front-to-back ratio.

Manuscript received October 20, 2008; revised June 10, 2009. First published November 06, 2009; current version published January 04, 2010 This work was supported by the TNO and by the NL Ministry of Defense in the framework of the research program Integrated Technology Mast Systems.

M. Pasian is with the Department of Electronics, University of Pavia, Pavia, Italy (e-mail: marco.pasian@unipv.it).

S. Monni, A. Neto, G. Gerini are with TNO Defence, Security and Safety, Den Haag 2597 AK, The Netherlands (e-mail: stefania.monni, andrea.neto, giampiero.gerini@tno.nl).

M. Ettorre was with TNO Defence, Security and Safety, Den Haag 2597 AK, The Netherlands. He is now with the Groupe Antennes and Hyperfréquences, Institut d'Electronique et de Télécommunications de Rennes (IETR), UMR CNRS 6164, Université de Rennes 1, 35042 Rennes Cedex, France (e-mail: mauro.ettorre@univ-rennes1.fr).

Digital Object Identifier 10.1109/TAP.2009.2036185
To overcome this problem, the most common approach consists in introducing a backing reflector. This backing reflector should be located at a distance of a small fraction of wavelength from the antenna if maximum gain is required, as outlined in [1]. However, a widely adopted choice is to place the reflector at a quarter wavelength distance from the antenna, as for example in [4]. Although this choice still allows obtaining a good antenna matching, with only a modest degradation of the achievable gain, the functioning of the backing reflector is optimal only in a relatively small frequency band [1]. Therefore, the improvement of the front-to-back ratio comes at the expense of the antenna bandwidth.

A way to tackle this problem is by introducing a frequency dependent backing reflector, obtained by combining a frequency selective surface (FSS) with a PEC ground plane. As outlined in [1], the FSS introduces an additional frequency band, resulting in a multi-band behavior of the backing reflector. Resistively loaded FSSs [5] and electronic bandgap structures (EBGs) [6] have already been proposed to obtain a wideband behavior. In [6], EBGs were designed to extend the bandwidth of a wideband connected array, which in the lower range of the operating frequency band was backed by an absorbing layer. In fact a backing metallic reflector for the lowest frequencies should have been located at a distance from the antenna plane in the order of half a meter. However, the use of resistive elements introduces about $3 \mathrm{~dB}$ of losses [1], [5], [6].

In this paper, the step to achieve good front-to-back ratios for a wide-angle scanning array antennas without resistive loading is attempted, taking as starting point a schematic design of a connected dipole array [7], [8]. In view of this, the paper represents the complementary side of a unique project aimed to wideband connected arrays backed by innovative ground planes. An alternative for the antenna could be offered by the arrays proposed in [1], which are very similar to connected arrays. In [1] the continuity of the electric current between array elements is obtained by means of capacitive loadings, instead of using physically connected dipoles as in [7], [8]. However, the designs proposed in [1] achieve the required wide bandwidth when one or more dielectric layers are introduced in front of the array. On the contrary, in this paper any dielectric layer is intentionally discarded, also to preserve the highest polarization purity [10].

The considered application foresees the integration of two frequency bands, one corresponding to the typical radar X-band, 8.50-10.50 GHz, and the other one corresponding to a Tactical Common Data Link (TCDL) system, 14.40-15.35 GHz. In this paper we consider the simplified case of $1 \mathrm{D}$ scanning in elevation up to $\pm 45^{\circ}$ and one linear polarization. A connected dipole array is backed by a combination of a ground plane and 


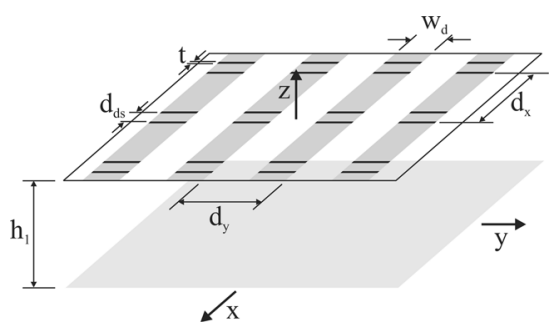

Fig. 1. 3-D view of the antenna structure with ground plane.

TABLE I

GeOMETRICAL Dimensions IN MM OF THE CONNECTED Dipole ARRAY Backed by a Single Metal Plate, Depicted in Fig. 1

\begin{tabular}{|c|c|c|c|c|c|}
\hline$w_{d}$ & $t$ & $d_{d s}$ & $d_{x}$ & $d_{y}$ & $h_{1}$ \\
\hline 1.5 & 0.5 & 3.1 & 8.3 & 8.3 & 8.3 \\
\hline
\end{tabular}

an FSS, which are designed to behave as perfect reflectors in two different frequency ranges. In particular, for lower frequencies, where the FSS is transparent for the impinging field, the backing reflector of the array is the real ground plane, whereas in the upper frequency range the FSS behaves as perfect reflector. A prototype of the backing structure consisting of the ground plane and the FSS has been manufactured. The magnitude of the reflection coefficient of the FSS alone and the phase of the reflection coefficient of the entire backing structure have been measured.

The Green's function (GF) and the active impedance of the structure composed by the antenna and the combined backing reflector have also been derived by extending the steps outlined in [11]. In the transmission line model of the entire structure, the FSS has been described by an equivalent network based on a modal representation in terms of spacial Floquet modes. The eventual GF provides physical insights in the problem and speeds up the antenna design and the optimization of the relative distance between the antenna, the FSS and the ground plane.

The paper is organized as follows. In Section II a connected dipole array backed by a conventional metallic ground plane is presented. This configuration appears not suitable to integrate the radar and TCDL bands. The solution proposed for the backing reflector, consisting of the FSS and the ground plane is presented in Section III. The measurements of a hardware demonstrator of the backing structure are shown in Section IV. The mathematical details to derive the active impedance of the complete antenna and the simulated results for the final structure are presented in Sections V and VI, respectively.

\section{Connected Dipole ARRAY Antenna With BaCKing GROUND PLANE}

The ultra-wide band antenna considered in this paper, a connected dipole antenna, and its complementary counterpart, the long-slot array antenna, have been extensively studied in [3], [4], [11] and [7], [8]. The antenna considered in this paper is shown in Fig. 1, with the parameters defined in Table I.

Such an antenna is composed by connected dipoles of width $w_{d}$, which are depicted in Fig. 1 as gray strips along the $x$ axis. The connected dipoles are excited by couples of $\delta$ - gap ports of width $t$ and relative separation $d_{d s}$, depicted in Fig. 1 as thin

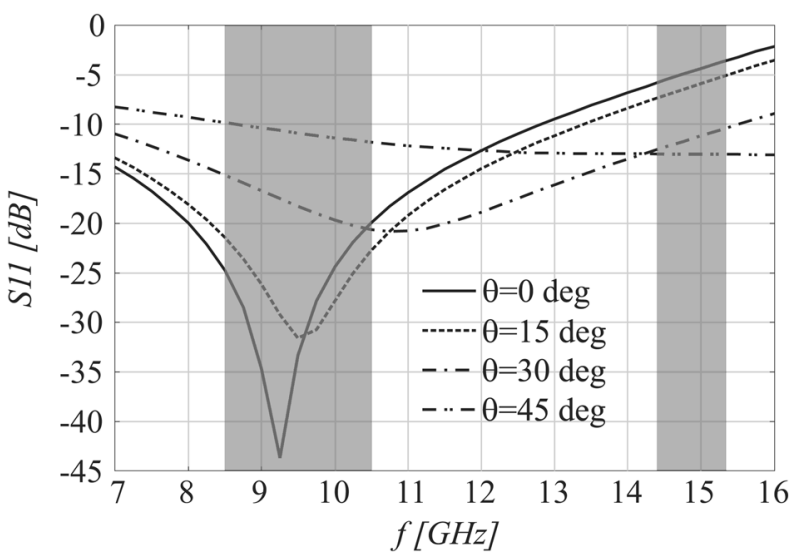

Fig. 2. Magnitude of the active reflection coefficient of the connected dipole array antenna with the ground plane. Gray strips indicate the considered operative bands.

black lines along the $y$ axis. The dimensions of the unitary cell are $d_{x}=d_{y}=0.415 \lambda_{\max }$, where $\lambda_{\max }$ is the free space wavelength at $f=15 \mathrm{GHz}$. The use of $\delta$ - gap ports, even if introduces some minor limitations from the simulation point of view, allows for effectively analyzing the array with no need of a detailed feeding line, which is out of the scope of the present work. The feeding lines would affect the behavior of the array as well as the behavior of the FSS, as shown for example in [12]. The first problem is accurately addressed in [8], [9], while the second problem, which is largely tied to technological aspects, working frequencies and materials will be considered in a later stage when results from both connected array and backing plane developments will be consolidated.

The distance $h_{1}$ between the antenna and the ground plane has been optimized at the value $8.3 \mathrm{~mm}$, trying to cover the entire 8.5-15.35 GHz band, for the complete scan range $\pm 45^{\circ}$. Fig. 2 shows the simulated active reflection coefficient [13], [14], for different scan angles in the H-plane (y-z plane), obtained with the commercial tool Ansoft HFSS [15]. It should be noted that only one linear polarization is required for the addressed application. Therefore, in this paper the scanning behavior of the antenna and the backing reflector will be investigated only for the H-plane. A complete evaluation of the scanning behavior of wideband connected arrays for both polarizations can be found in [8]. Considering $-10 \mathrm{~dB}$ as threshold limit for the active reflection coefficient, it is evident from Fig. 2 that the ground plane solution fulfils the desired requirements only in the radar frequency band, presenting very poor performances in the TCDL band.

\section{Frequency Selective Surface Design}

The previous section clearly showed that the connected array with a conventional metal ground plane is not suitable to simultaneously cover the radar and the TCDL bands. As anticipated in the introduction of this paper, this limitation can be overcome by sandwiching an FSS between the array and the metal ground plane.

In particular, in this case, the FSS should separate the frequency bands allocated for naval radar operation, 8.50-10.50 


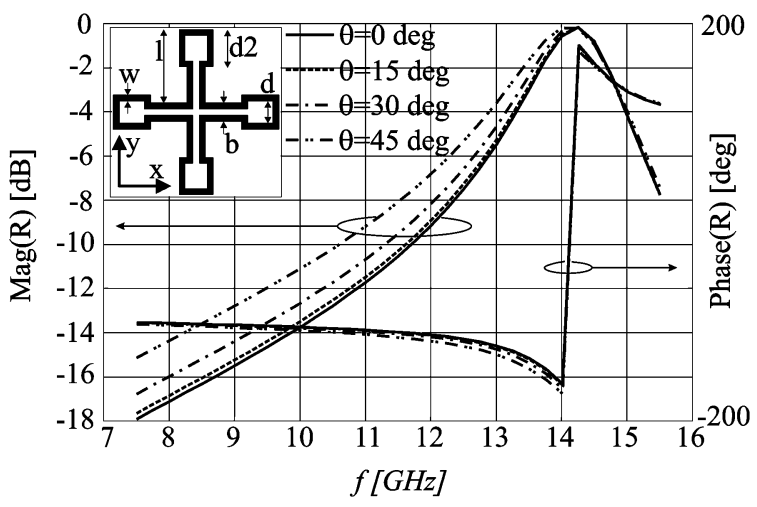

Fig. 3. Magnitude and phase of the reflection coefficient of the FSS for different angles of incidence for TE-polarized plane wave incidence.

TABLE II

Geometrical Dimensions in MM of the Backing Plane Composed By THE FSS AND THE GROUND Plane DePICTED IN FIGS. 3 AND 4

\begin{tabular}{|c|c|c|c|c|}
\hline$w$ & $l$ & $b$ & $d$ & $d 2$ \\
\hline 0.2 & 2.245 & 0.6 & 0.7 & 1.2 \\
\hline
\end{tabular}

$\mathrm{GHz}$ and for the TCDL, $14.40-15.35 \mathrm{GHz}$, thus providing a reflection plane for the higher frequency range and being practically transparent for the lower band, where the metallic ground plane is in charge of the reflection. On top of that, both applications ask for a wide-scan capability, up to $\pm 45^{\circ}$ in elevation.

In order to achieve the desired behavior the FSS should be transparent in the $8.50-10.50 \mathrm{GHz}$ frequency range and reflective in the $14.40-15.35 \mathrm{GHz}$ frequency range. For this purpose, a common approach is to print metallic patches, resonating at the higher frequency band, on a dielectric substrate. The adopted solution is based on single-layer structures with a foam substrate, which is assumed to be electrically equivalent to air. This choice minimizes the manufacturing effort and the losses. The severe constraints imposed by the wide angle scanning and steep roll-off required to properly separate the two bands dominated the design.

The element shape selected for the FSS is the four-legged dipole. This kind of element exhibits a good performance against angular variation and, since the total element length can be kept quite small [16], it allows a very packed lattice, with a further gain in angular independence. On the other hand, the more packed the elements are the less steep the roll-off is, due to the capacitive coupling between the elements. For this reason a proper tradeoff had to be performed.

The chosen final FSS element is depicted in the inset of Fig. 3 and its dimensions are given in Table II. The lattice periodicity, $d_{x}=d_{y}=8.3 \mathrm{~mm}$, equal to that of the connected array, allows good angular independence, also avoiding grating lobes in the considered band. The four-legged dipoles are capacitively loaded to achieve the required roll-off needed to separate the radar and the TCDL bands, while preserving the FSS resonance frequency.

The magnitude and phase of the FSS reflection coefficient, simulated with the commercial software Ansoft Designer [17] are reported in Fig. 3. Note that the phase of the reflection coefficient is evaluated at the FSS plane itself. A uniform plane
TABLE III

GEOMETRICAL DimENSIONS IN M OF THE CONNECTED Dipole ARRAY BACKED By the FSS AND THE GRound Plane, DePiCted IN FIG. 9

\begin{tabular}{|c|c|c|c|c|c|c|}
\hline$w_{d}$ & $t$ & $d_{d s}$ & $d_{x}$ & $d_{y}$ & $h_{1}$ & $h_{2}$ \\
\hline 1.5 & 0.5 & 3.1 & 8.3 & 8.3 & 6.5 & 2.3 \\
\hline
\end{tabular}

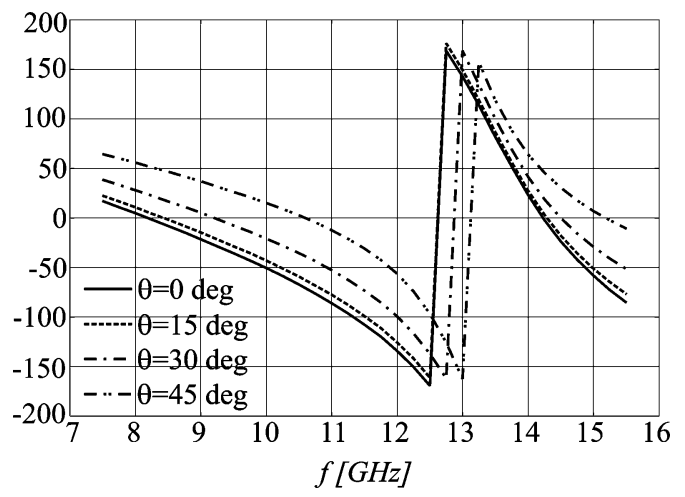

Fig. 4. Phase of the reflection coefficient of the FSS backed by a ground plane for different angles of incidence for TE-polarized plane wave incidence.

wave polarized along the $x$-axis (TE polarization), impinging at different angles on the $y-z$ plane, was considered as excitation, consistently with the analysis results reported in Section II for the connected array. It can be observed that the FSS does not exactly resonate at the center of the TCDL band. Because of the proximity and thus the interaction between the FSS itself and the ground plane, the FSS design had to be tuned to optimize the phase behavior of the complete backing reflector (FSS + ground plane).

Then, the optimal distances between the ground plane and the FSS, and between the FSS itself and the antenna plane had to be determined. In first approximation the FSS and the ground plane should be placed at a distance of $\lambda / 4$ from the antenna plane, calculated at central frequencies of the two considered frequency bands. In this way, a constructive interference between the direct field generated by the antenna and the one coming back from the FSS or ground plane would be obtained.

On the other hand, the interaction between the ground plane and the FSS, which are very close to each other in terms of wavelength, has a significant impact on the final results. For this reason a fast full-wave method was used to accurately calculate the best distances, as described in Section V. The obtained values, $h_{1}$ and $h_{2}$, (see Fig. 9) are reported in Table III

Fig. 4 reports the phase of the reflection coefficient exhibited by the entire structure composed by the FSS and the ground plane, for scanning angles up to $45^{\circ}$. The phase response depicted in Fig. 4 is evaluated at the optimized distance $h_{1}$ of 6.5 $\mathrm{mm}$ between FSS and antenna plane. The magnitude of the reflection coefficient is not reported because it is always equal to $0 \mathrm{~dB}$, due to the presence of the perfectly metallic ground plane.

It can be observed that the constructive interference, which is indicated by a reflected phase equal to $0^{\circ}$, is roughly achieved at the centers of the two separate operating bands.

Although the chosen FSS element is geometrically symmetric, the behavior is not exactly the same for the two orthogonal polarizations [16]. Therefore, for applications in 


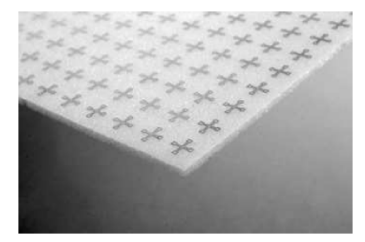

Fig. 5. Close-up view of the manufactured FSS.

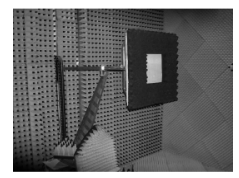

Fig. 6. Photograph of the mechanical support.

which dual linear or circular polarization is required, the design of the backing reflector should be further optimized.

\section{MEASUREMENTS}

The backing plane design was carried out considering only foam as substrate, but manufacturing constraints required the use of an extra dielectric support, as depicted in the inset of Fig. 8. The original design was tuned to account for the different equivalent dielectric constant. The FSS was printed on a $60 \times 60 \mathrm{~cm}$ thin dielectric substrate from Rogers, LG4002 $\left(\epsilon_{r 1}=2.89, t_{1}=0.075 \mathrm{~mm}\right)$. This layer was glued on a foam from Rohacell $\left(\epsilon_{r 3}=1.06, t_{3}=2.2 \mathrm{~mm}\right)$ by means of an adhesive film from Arlon, CuClad $6250\left(\epsilon_{r 2}=2.32, t_{2}=0.039\right.$ $\mathrm{mm})$. A metallic plate was then fixed at the bottom of the foam, finalizing the manufacturing of the complete backing structure. A close-up photograph of the FSS without the metallic plate is shown in Fig. 5. Two measurement setups were considered: one for the FSS alone, before attaching the ground plane, and the other one for the complete backing structure. Both measurements took place in an anechoic chamber using two standard wideband double-ridge horn antennas, in a quasi-monostatic configuration for broadside measurements and in a bistatic configuration for other angles of incidence.

The position of the backing plane and of the FSS were controlled thanks to a mechanical construction consisting of a mast, two vertical and horizonal poles, with elevation and azimuth adjustable joints, and a square frame (Fig. 6). The mast was specifically designed for microwave measurements showing a reflection coefficient better than $-30 \mathrm{~dB}$. The main part of the entire support consisted of a square frame where the backing plane could be precisely slid in. This frame was connected to the rest of the support by means of two vertical and two horizontal arms bent behind the frame, to reduce their contribution to the reflection. Thanks to this arrangement most of the reflection was caused by parts located at about $30 \mathrm{~cm}$ away from the frame. Therefore, such reflection contributions could be removed from the measurement by applying a proper time gate in the network analyzer. Absorbing material was placed around the frame to reduce edge effects and to hide the supporting structure mentioned before. The alignment between the backing plane and the horn antennas was controlled by means of laser beams and optimized by adjusting the elevation and azimuth joints.
When measuring the reflection phase, even small inaccuracies may translate to several degrees of errors. For this reason, a particular measurement procedure was applied to retrieve the phase of the reflection coefficient of the backing plane under test, minimizing errors due to edge effects, to the fact that the electromagnetic field generated by the horn antenna is not a uniform plane wave and due to deviations of the horn antenna phase center position with the frequency. A conventional metallic reflector, with dimension and position exactly equal to the ones of the backing plane under test, was used as reference. The phase of the reflection coefficient given by the perfect metallic plate recorded by the network analyzer can be written as

$$
\alpha=2 k d-\pi+E
$$

where $\alpha$ is the phase recorded by the horn antenna, $d$ is the distance between the horn antenna and the metallic plate, $\pi$ is the analytical reflection phase given by an infinity perfectly conducting plate and $E$ takes into account any other error.

Then the backing structure was measured and a similar equation holds

$$
\beta=2 k d+\gamma+E
$$

where $\beta$ is the phase recorded by the horn antenna and $\gamma$ is the reflection phase given by the backing structure. The error $E$ was assumed to be equal in both cases.

Now it is possible to obtain the requested value $\gamma$, substituting (1) into (2)

$$
\gamma=\beta-\alpha-\pi
$$

Of course, after this procedure, it is still necessary to report the retrieved reflection phase given by the backing structure to the plane where the antenna array will be placed, at a distance of $h_{1}=6.5 \mathrm{~mm}$ as shown in the previous section.

A similar reference procedure was also followed to calibrate the response of the FSS alone. In this case the magnitude of the reflection coefficient was obtained considering as reference the reflection coefficient of the complete backing structure. In fact, the latter has a magnitude of the reflection coefficient equal to 0 $\mathrm{dB}$ at any frequency.

The experimental results are shown in Figs. 7 and 8, where they are compared with the results obtained from simulations for incidence angles of $0^{\circ}, 30^{\circ}$ and $45^{\circ}$. The agreement between theory and measurement is very good, both for the magnitude and the phase response. In particular, Fig. 7 shows an excellent agreement close to the resonance region of the FSS, with some minor differences at lower frequencies, where the FSS is almost invisible and therefore the mechanical structure behind it may have affected the measurement. At the highest frequency range a slight shift can be observed, which is probably due to some small unpredictable variations of the features of the dielectric support during the manufacturing process. Fig. 8 shows the phase response of the complete backing plane, calculated at the antenna plane as described in the inset of Fig. 8 itself. The agreement is very good for all considered angles, despite the extremely high 


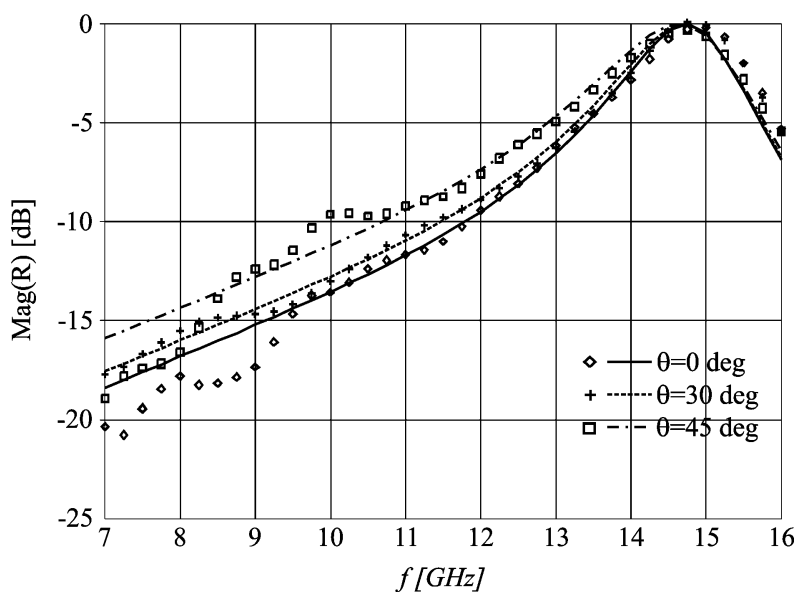

Fig. 7. Measured magnitude of the reflection coefficient of the FSS for different angles of incidence for TE-polarized plane wave incidence. Lines refer to HFSS results while symbols to measurement.

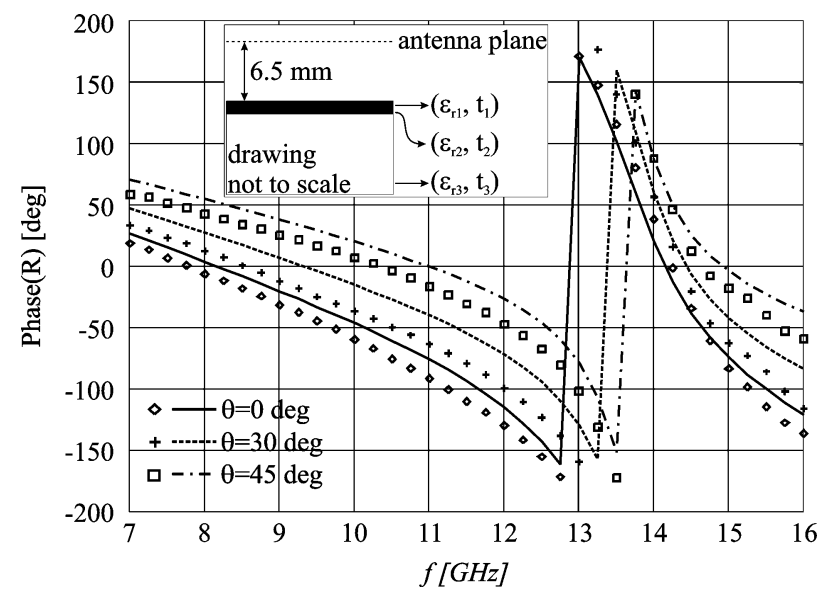

Fig. 8. Measured phase of the reflection coefficient of the FSS backed by a ground plane for different angles of incidence for TE-polarized plane wave incidence. Lines refer to HFSS results while symbols to measurement.

accuracy required for such measurement and despite the rate of the phase variation in proximity of the FSS resonance. Small discrepancies at lower frequency are probably due to a differences between the actual and nominal values of the dielectric constant and of the thickness of the dielectric support. Once a sufficient knowledge of the impact of the fabrication process on the relevant parameters of the structure (i.e., dielectric constants and layer thicknesses) will be acquired, as part of a standard engineering development, the minor shifts observed in the measurements could be tuned out by slightly adjusting the FSS geometry and the relative separations between FSS, ground plane and antenna.

\section{2-D CONNECTED ARRAY: ACTIVE IMPEDANCE IN THE PRESENCE OF THE FSS}

The active impedance of connected dipole arrays backed by ground plane [8] and FSS can be derived by extending the steps outlined in previous works [3] and [11], where the GF and the

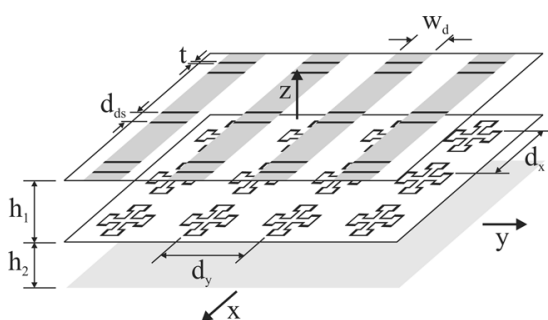

Fig. 9. 3-D view of the antenna structure with the FSS and the ground plane.

active impedance for a 2-D long slot connected array was found. The complete structure is shown in Fig. 9.

As demonstrated in [8] the spectral representation of the input admittance of an array of dipoles with a double feed in each cell and in the presence of a ground plane as backing reflector can be expressed analytically as

$$
y=\frac{k_{0}}{\zeta_{0}} \frac{d_{x}}{d_{y}} \sum_{m_{x}=-\infty}^{\infty} \frac{\cos ^{2}\left(k_{k x m} d_{d s} / 2\right) \operatorname{sinc}^{2}\left(k_{k x m} t / 2\right)}{\left(k_{0}^{2}-k_{x m}^{2}\right) \sum_{m_{y}=-\infty}^{\infty} \frac{J_{0}\left(k_{y m} w_{d} / 2\right)}{k_{z m}\left(1-j \cot \left(k_{z m} h_{d}\right)\right)}} .
$$

where $d_{x}$ and $d_{y}$ are the periodicities of the array, $d_{d s}$ is the distance between the two feeds inside each unit cell, $k_{0}$ is the free-space propagation constant, $J_{0}$ is the zeroth order Bessel function and $k_{x m}$ and $k_{y m}$ are the $\left(m_{x}, m_{y}\right)$ Floquet mode propagation constants along $x$ and $y$. Please refer to [11] for a complete definition of the functions used in the previous and following expressions.

From the formalism described in depth in [11] the same expression can be recognized as the explicit representation of

$$
y=\frac{d_{x}}{d_{y}} \sum_{m_{x}=-\infty}^{\infty} \frac{-\cos ^{2}\left(k_{k x m} d_{d s} / 2\right) \operatorname{sinc}^{2}\left(k_{k x m} t / 2\right)}{\sum_{m_{y}=-\infty}^{\infty} G_{x x}^{b r}\left(k_{x m}, k_{y m}\right) J_{0}\left(k_{y m} w_{d} / 2\right)} .
$$

The electromagnetic field in the zone of space below the connected dipoles is entirely represented by $G_{x x}^{b r}$, where $x x$ indicates that the GF provides the $x$ component of the electric field radiated by the $x$ component of the electric current, and $b r$ stands for backing reflector. Equating (4) and (5) it is apparent that

$$
G_{x x}^{b r}\left(k_{x}, k_{y}\right)=\frac{-\zeta_{0}}{2 k_{0}} \frac{\left(k_{0}^{2}-k_{x}^{2}\right)}{k_{z}}\left(1-e^{-2 j k_{z} h_{d}}\right)
$$

and thus, this structure's spectral GF is analytically known. Readers are reminded that each of the spectral components $\left(k_{x m x}, k_{y m y}\right)$ of any GF represents the response to a combination of TE and TM plane waves. For instance

$$
G_{x x}\left(k_{x m}, k_{y m}\right)=\frac{-1}{k_{x m}^{2}+k_{y m}^{2}}\left(V_{\mathrm{TM}} k_{x m}^{2}+V_{\mathrm{TE}} k_{y m}^{2}\right)
$$




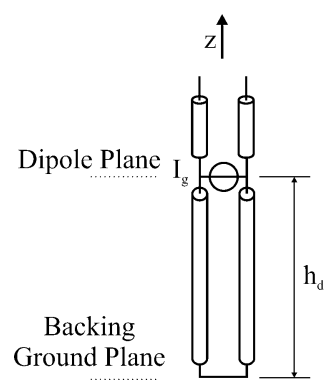

Fig. 10. Generic transmission line model for a Floquet mode in presence of the connected dipole array backed with a ground plane. The transmission line model is the same for TE and TM modes once the proper definition of the characteristic line impedance is chosen. $I_{g}$ is a unit current generator.

where $V_{\mathrm{TM}}$ and $V_{\mathrm{TE}}$ are the amplitudes of electric fields of TM and TE plane waves respectively, in the geometrical environment that the GF represents. In the case of laterally infinite layered structures the response for each plane wave is analytically known because it only depends on the $z$ dimensions, the thickness of the layers. Thus this dependency is represented by two equivalent transmission lines, TE or TM, as in Fig. 10, where the sources are unit current generators. It is simple to show that the solution of these transmission lines combined as in (7) gives rise to (6)

When an FSS is inserted between the dipoles and the ground plane, the general GF representation for the input admittance in (7) is still valid by substituting $G_{x x}^{b r}$ with $G_{x x}^{f s s+b r}$. However in this case $G_{x x}^{f s s+b r}$ is not analytically known. The FSS loads each of the plane waves of the spectral domain GF in ways which depend also on the transverse details of the FSS element geometry, (e.g., lengths and width of the legs of the four-legged loop element in the present case). This information is critical to the definition of the resonance frequency of the FSS. The FSS loading impact can be accounted for by equivalent impedance loads in parallel to the Floquet mode equivalent transmission lines representing the propagation in the otherwise transversally infinite layered cross sections. Several full wave techniques can be applied to evaluate these equivalent impedance loads when only the main Floquet mode $(m=n=0)$ is accounted for.

The overall mono-mode equivalent network valid for each of the plane wave components, TE and TM, of the spectral GF also accounting for the FSS is shown in Fig. 11, where $Z_{\mathrm{FSS}}$ represents the equivalent FSS impedance load for the main Floquet mode. As apparent from (4) and (5), the impedance load that goes in parallel to the transmission line needs to be evaluated only for the plane wave directions associated to $k_{x m}$ and $k_{y m}$, i.e., $\theta_{m x, m y}$ and $\phi_{m x, m y}$, defined by inverting the relations

$$
\begin{aligned}
& k_{x m}=k_{0} \sin (\theta) \cos (\phi)-\frac{2 \pi m}{d_{x}} \\
& k_{y n}=k_{0} \sin (\theta) \sin (\phi)-\frac{2 \pi n}{d_{y}} .
\end{aligned}
$$

If not only the fundamental mode but also higher order Floquet modes contribute to the interaction between the composite ground plane and antenna that is they constitute accessible

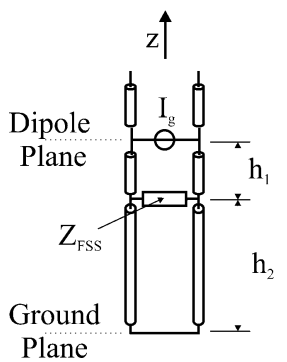

Fig. 11. Equivalent transmission line model of the planar structure of Fig. 9 for the main FW's mode. $h_{1}$ and $h_{2}$ are respectively the distance of the FSS from the connected dipole array and from the backing ground plane.

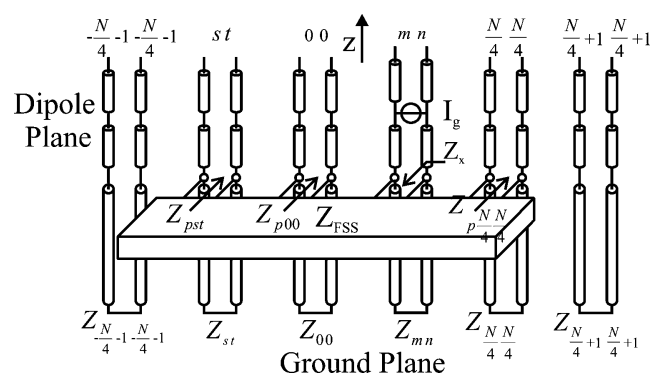

Fig. 12. Equivalent transmission line model of the planar structure of Fig. 9.

modes according the terminology of [18], the equivalent circuit becomes a multimode network. If $\mathrm{N}$ accessible modes are included, an accurate description of the $k_{x m}, k_{y m}$ component of the GF is obtained by solving the equivalent network problem depicted in Fig. 12. This is not conceptually different with respect to the much simpler equivalent circuit in Fig. 11 but it is more accurate, should the distance between the ground plane and the FSS become extremely small in terms of wavelength.

Note that the relevance of this multimode extension to the present case appears to be limited. On one hand, only at very low frequencies the distance between FSS and ground plane would be such that higher order modes have to be included in the field representation. On the other hand, at these frequencies the FSS would be transparent and not significantly affect the behavior of the overall structure.

Once the GF (4) is known, it is possible to study the final antenna structure shown in Fig. 9, where the FSS is the one designed in Section III. The only two parameters remaining to the designer $h_{1}$ and $h_{2}$ can be optimized very efficiently by using the GF that also includes the effect of the FSS. The dimensions of the final structure are reported in Table III.

\section{ARRAY PERFORMANCES}

Fig. 13 shows a comparison of the results concerning the active reflection coefficient for scanning on the $\mathrm{H}$-plane of the antennas, obtained with Ansoft HFSS and with the method described in the previous section. Good agreement is achieved for all scan angles. For our method, only the fundamental modes were considered. After several simulations performed including a larger number of Floquet modes, it was observed that the fundamental modes were sufficient to describe the discontinuity introduced by the FSS. The active reflection coefficient depicted 


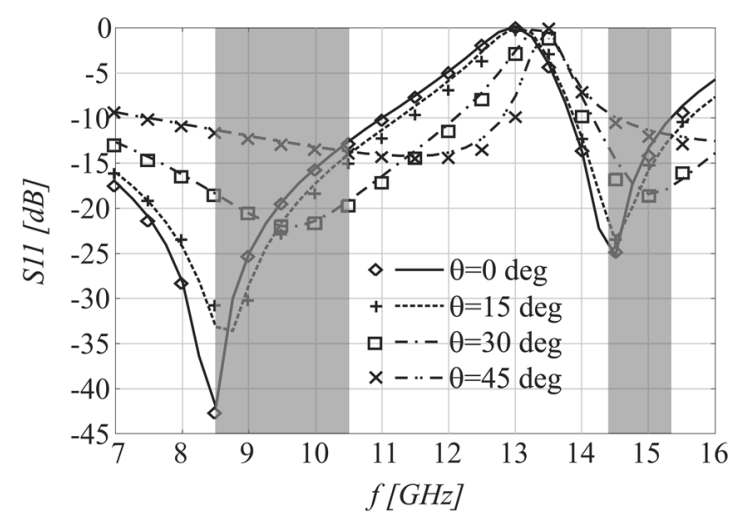

Fig. 13. Magnitude of the active reflection coefficient of the connected dipole array antenna with the FSS and the ground plane. Lines refer to HFSS results while symbols to the in-house method.

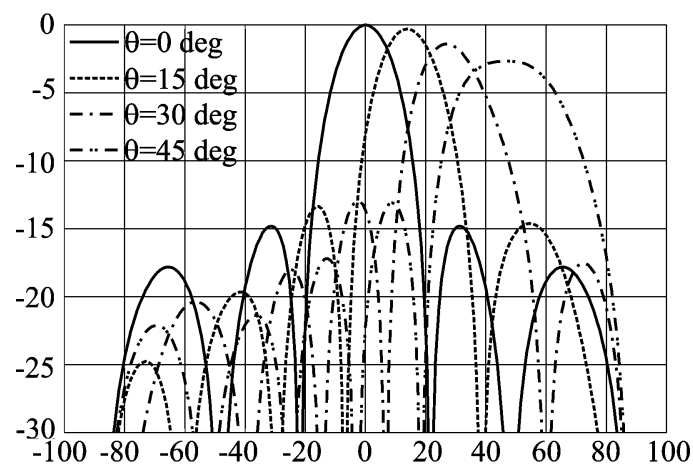

Fig. 14. Radiation patterns on the H-Plane for different scan angles at the central frequency of the radar band, $f=9.5 \mathrm{GHz}$, for the connected dipole array antenna backed by the ground plane and FSS. The pattern are normalized to the maximum value for broadside radiation.

in Fig. 13 was obtained normalizing to a feeding port impedance of $400 \mathrm{Ohm}$.

A good matching below the threshold value of $-10 \mathrm{~dB}$ is achieved in the entire scan range in both the radar and TCDL bands, showing the possibility to extend the operational bands of the connected array by using the proposed backing structure.

It is also evident that such a configuration for a backing reflector, which relies on an FSS on the top of standard metallic plate, always exhibits a multi-band behavior instead of a unique wideband operative frequency region. The basic reason for that is related to the fact that each backing plate composing the entire reflector (i.e., the FSS and the metallic ground plane) constructively reflects the field generated by the array (i.e., the phase of the reflection coefficient equal to $0^{\circ}$ ) for one precise frequency, given the distance from the antenna and the backing structure. Thus, since the phase of the reflection coefficient is a continuous and periodic function and since the sign of its derivative is constant with the frequency, in order to connect the two frequencies where the reflection phase is $0^{\circ}$ the function must exhibits a passage through the $\pm 180^{\circ}$ region, which correspond to a disruptive interference. This aspect was already observed in [1].

For the sake of completeness the normalized gain pattern on the H-plane at the central frequency of the radar and TCDL bands, obtained by using Ansoft HFSS, are shown in Figs. 14

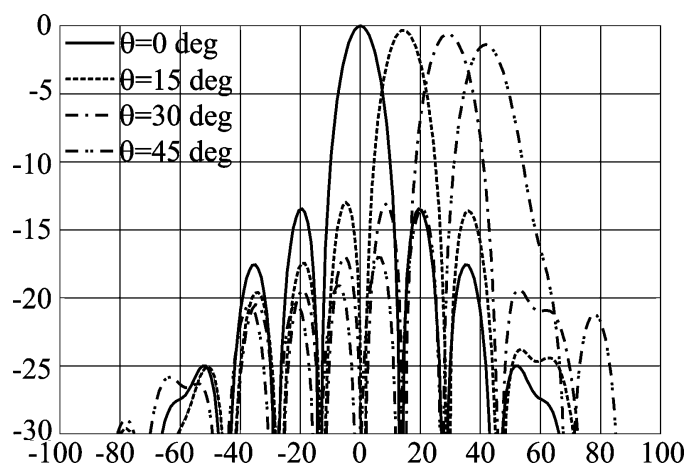

Fig. 15. Radiation patterns on the H-Plane for different scan angles at the central frequency of the TCDL band, $f=15 \mathrm{GHz}$, for the connected dipole array antenna backed by the ground plane and FSS. The pattern are normalized to the maximum value for broadside radiation.

and 15 respectively, for different scan angles and considering the case of a $10 \times 10$ element finite array.

\section{CONCLUSION}

This paper has presented a method for the extension of the operating bandwidth toward higher frequencies of a wideband antenna on top of a metallic reflector. This is achieved by sandwiching a Frequency Selective Surface between the antenna and the ground plane. In particular, the design of a connected dipole array that integrates the functionalities of radar and Tactical Common Data Link, has been presented. A prototype of the backing reflector made by the FSS and the ground plane has been manufactured and successfully tested, showing very good agreement between predictions and measurement. Besides, the procedure to derive the active impedance for the complete antenna structure has been illustrated. The results have been compared with the ones obtained through commercial tools showing an excellent agreement.

It may be noted that the performance achievable from the connected dipole array backed by the composite reflector are significantly dependent on the scan angle. Thus, the distances between the reflecting planes and the antenna $h_{1}$ and $h_{2}$, should always be accurately tuned for optimal performance of the full angular range. To this purpose, the availability of the active Green's function presented in Section V greatly accelerates the design optimization.

\section{ACKNOWLEDGMENT}

The authors wish to thank F. Nennie, P. Kuivenhoven and M. Bruijn for their crucial contributions to the design and manufacture of the mechanic support and to the measurement campaign.

\section{REFERENCES}

[1] B. A. Munk, Finite Antenna Arrays and FSS. New York: Wiley, 2003, ch. 6 , pp. 181-213.

[2] J. J. Lee, S. Livingston, and R. Koenig, "A low-profile wideband (5:1) dual-pol array," IEEE Antennas Wireless Propag. Lett., vol. 2, pp. 46-49, Dec. 2003.

[3] A. Neto and J. J. Lee, "Infinite bandwidth long slot array antenna," IEEE Antennas Wireless Propag. Lett., vol. 3, pp. 75-78, Dec. 2005. 
[4] J. J. Lee, S. Livingston, R. Koenig, D. Nagata, and L. L. Lai, "Compact light weight UHF arrays using long slot apertures," IEEE Trans. Antennas Propag., vol. 54, no. 7, pp. 2009-2015, Jul. 2006.

[5] Y. E. Erdemli, K. Sertel, R. A. Gilbert, D. E. Wright, and J. L. Volakis, "Frequency-selective surfaces to enhance performance of broad-band reconfigurable arrays," IEEE Trans. Antennas Propag., vol. 50, no. 12, pp. 1716-1724, Dec. 2002.

[6] J. M. Bell, M. F. Iskander, and J. J. Lee, "Ultrawideband hybrid EBG/ ferrite ground plane for low-profile array antennas," IEEE Trans. Antennas Propag., vol. 55, no. 1, pp. 4-12, Jan. 2007.

[7] D. Cavallo, A. Neto, G. Gerini, and G. Toso, "On the potentials of connected slots and dipoles in the presence of a backing reflector," presented at the 30th ESA Antenna Workshop, Noordwijk, The Netherlands, May 27-30, 2008.

[8] A. Neto, D. Cavallo, G. Gerini, and G. Toso, "Scanning performances of wide band connected arrays in the presence of a backing reflector," IEEE Trans. Antennas Propag., vol. 57, no. 10, pp. 3092-3102, Oct. 2009, part 2.

[9] A. Neto, D. Cavallo, and G. Gerini, "Common mode, differential mode and baluns: The secrets," presented at the 5th ESA Workshop on Millimetre Wave Technology and Applications and 31st ESA Antenna Workshop, Noordwijk, The Netherlands, May 18-20, 2009.

[10] A. Hoorfar, K. Gupta, and D. Cahng, "Cross polarization level in radiation from microstrip dipole antenna," IEEE Trans. Antennas Propag., vol. 36, no. 9, pp. 1197-1203, Sep. 1988.

[11] A. Neto and J. J. Lee, "Ultrawideband properties of long slot arrays," IEEE Trans. Antennas Propag., vol. 54, no. 2, pp. 534-543, Feb. 2006.

[12] S. G. Hay and J. D. O'Sullivan, "Analysis of common-mode effects in a dual-polarized planar connected-array antenna," Radio Sci., vol. 43, Dec. 2008, RS6S04.

[13] R. C. Hansen, Phased Array Antennas. New York: Wiley, 1998.

[14] D. M. Pozar, "The active element pattern," IEEE Trans. Antennas Propag., vol. 42, no. 8, pp. 1176-1178, Aug. 1994.

[15] Ansoft HFSS Version 10.0, 1984-2007 Ansoft Corporation.

[16] B. A. Munk, Frequency Selective Surfaces: Theory and Design. New York: Wiley, 2000.

[17] Ansoft Designer Version 3.0.0, 1984-2007 Ansoft Corporation.

[18] S. Monni, G. Gerini, A. Neto, and A. G. Tijhuis, "Multimode equivalent networks for the design and analysis of frequency selective surfaces," IEEE Trans. Antennas Propag., vol. 55, no. 10, Oct. 2007.

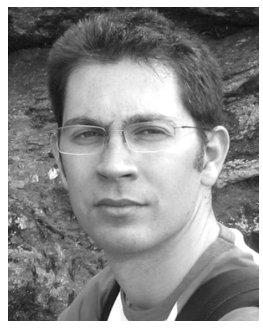

Marco Pasian (S'04-M'08) was born in 1980. He received the M.S. degree (summa cum laude) in electronic engineering and the $\mathrm{Ph} . \mathrm{D}$. degree in electronics and computer science from the University of Pavia, Pavia, Italy, in 2005 and 2009, respectively.

$\mathrm{He}$ is currently a Postdoctoral Researcher in the Department of Electronics, University of Pavia. His main research interests are periodic structures, antennas and microwave devices for space and defence applications. In 2004, he was a trainee at the European Space Agency, Darmstadt, Germany. In 2005, he was with Carlo Gavazzi Space, Milano, Italy, as a System Engineer. In 2008, he was a Guest Scientist at TNO, Defence, Security and Safety, The Hague, The Netherlands.

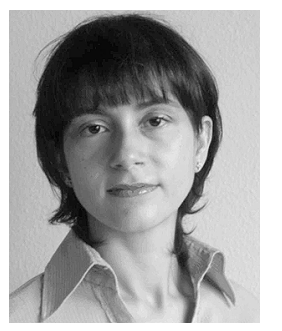

Stefania Monni (S'01-M'06) received the M.Sc degree (summa cum laude) in electronic engineering from the University of Cagliari, Italy, in 1999 and the Ph.D. degree in electronic engineering from the Technical University of Eindhoven, The Netherlands, in 2005.

In 1999 and 2000, she worked at the Radio Frequency System Division, European Space Research and Technology Centre (ESA-ESTEC) as an undergraduate and graduate trainee, respectively. From 2001 until 2005, she carried out her Ph.D. research at the Netherlands Organization for Applied Scientific Research (TNO), The Hague, The Netherlands, where she is currently employed. Her main research interests concern analysis and design techniques for phased array antennas and frequency selective surfaces, wide band antennas and digital beam forming for active and passive radars.

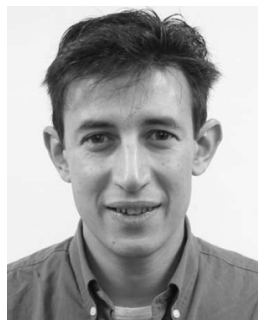

Andrea Neto (M'00) received the Laurea degree (summa cum laude) in electronic engineering from the University of Florence, Italy, in 1994 and the $\mathrm{Ph} . \mathrm{D}$. degree in electromagnetics from the University of Siena, Italy, in 2000.

Part of his Ph.D. was developed at the European Space Agency Research and Technology Center, Noordwijk, The Netherlands, where he worked for the Antenna Section for over two years. In the years 2000 to 2001 , he was a Postdoctoral Researcher at the California Institute of Technology, Pasadena, working for the Sub-mm wave Advanced Technology Group. Since 2002, he has been a Senior Antenna Scientist at TNO Defence, Security and Safety, The Hague, The Netherlands. His research interests are in the analysis and design of antennas, with emphasis on arrays, dielectric lens antennas, wide band antennas and EBG structures.

Dr. Neto was co-recipient of the H. A. Wheeler award for the Best Applications Paper of 2008 in the IEEE TRANSACTIONS ON ANTENNAS AND PROPAGATION. He presently serves as Associate Editor of the IEEE ANTENNAS AND WIRELESS PROPAGATION LETTERS.

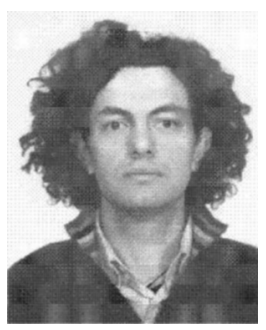

Mauro Ettorre (M'08) was born in Tricarico, Matera, Italy, in 1979. He received the Laurea degree (summa cum laude) in telecommunication engineering and the Ph.D. degree in electromagnetics from the University of Siena, Italy, in 2004 and 2008, respectively.

During his master degree studies he spent five months at the Technical University of Denmark (DTU), Lyngby, Denmark. Part of his Ph.D. has been developed at the Defence, Security and Safety Institute of the Netherlands Organization for Applied Scientific Research (TNO), The Hague, The Netherlands, and where afterwards he worked as a Researcher. Currently, he is a Postdoctoral Fellow at the Institut d'Electronique et de Télécommunications de Rennes (IETR), Université de Rennes 1, France, working for the Groupe Antennes \& Hyperfréquences. His research interests include the analysis and design of leaky-wave antennas, periodic structures and electromagnetic band-gap structures.

Dr. Ettorre received the Young Antenna Engineer Prize during the 30th ESA Antenna Workshop 2008, in Noordwijk, The Netherlands.

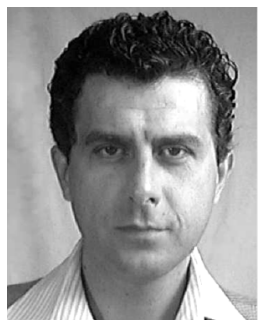

Giampiero Gerini (M'92-SM'08) received the M.Sc. degree (summa cum laude) and the Ph.D. degree in electronic engineering from the University of Ancona, Italy, in 1988 and 1992, respectively.

From 1992 to 1994, he was an Assistant Professor of Electromagnetic Fields at the University of Ancona. From 1994 to 1997, he was a Research Fellow at the European Space Research and Technology Centre (ESA-ESTEC), Noordwijk, The Netherlands, where he joined the Radio Frequency System Division. Since 1997, he has been with the Netherlands Organization for Applied Scientific Research (TNO), The Hague, The Netherlands. At TNO Defence Security and Safety, he is currently Chief Senior Scientist of the Antenna Unit in the Transceiver Department. In 2007, he was appointed part-time Professor in the Faculty of Electrical Engineering of the Eindhoven University of Technology, The Netherlands, with a Chair in Novel Structures and Concepts for Advanced Antennas. His main research interests are phased arrays antennas, electromagnetic bandgap structures, frequency selective surfaces and integrated antennas at microwave, millimeter and sub-millimeter wave frequencies. The main application fields of interest are radar, space and telecommunication systems.

Prof. Gerini was co-recipient of the 2008 H. A. Wheeler Applications Prize Paper Award of the IEEE Antennas and Propagation Society. In 2008, he was also co-recipient of the Best Innovative Paper Prize of the European Space Agency 30th Antenna Workshop. 